\title{
Pacific
}

Journal of

Mathematics

\section{EQUIVARIANT NIELSEN NUMBERS}

\section{Peter N-S Wong}

Volume $159 \quad$ No. 1 


\title{
EQUIVARIANT NIELSEN NUMBERS ${ }^{1}$
}

\section{Peter Wong}

\begin{abstract}
In this paper, we introduce equivariant Nielsen type numbers which estimate the minimal number of fixed orbits and fixed points of a $G$ map $f: X \rightarrow X$ in the $G$-homotopy class of $f$. As an application, we relate the equivariant Nielsen theory to the Nielsen theory for iterates of maps.
\end{abstract}

1. Introduction. Let $f: X \rightarrow X$ be a self map of an ENR $X$ so that the set of fixed points Fix $f$ is compact. In topological fixed point theory, the fixed point index $I_{f}$ ([D2]) is an algebraic count of the number of fixed points of $f$ so that $I_{f} \neq 0$ implies Fix $f \neq \varnothing$. When $X$ is compact, this algebraic count is given by the Lefschetz number $L(f)$ which can be expressed as a trace. However $L(f)$ does not usually give much information about the size of Fix $f$. A more subtle invariant $N(f)$, namely the Nielsen number of $f$, gives a lower bound for the minimal number of fixed points of maps in the homotopy class of $f$. In many situations, $N(f)$ is a sharp lower bound (e.g. when $M$ is a compact connected manifold of dimension $\geq 3)$.

Fixed point theory can be generalized to the study of periodic points, i.e., fixed points of $f^{n}=f \circ \cdots \circ f$. In [D3], Dold established a combinatorial relation among the fixed point indices of iterates of $f$. It was shown by Komiya [K] that Dold's relation can be derived from a similar congruence relation on the fixed point indices of equivariant maps. Nielsen fixed point theory was generalized to iterates of maps by Jiang [J], Heath-Piccinini-You [HPY], Heath-You [HY] and some earlier work of Halpern. The objective of this paper is to develop an equivariant Nielsen theory for $G$-maps. As an application, we indicate how the equivariant theory generalizes the periodic point theory in a similar fashion as [K] extends [D3].

For background in Nielsen fixed point theory, we refer the reader to $[\mathbf{B r}]$ and $[\mathbf{J}]$. For equivariant topology, the basic references are $[\mathbf{B}]$ and [tD]. This paper is organized as follows.

${ }^{1}$ The results in this paper were announced at the Special Session on Nielsen Fixed Point Theory in the Pre-Congress Topology Conference held at the University of Hawaii, August 12$18,1990$. 
In $\S 2$, we define equivariant Nielsen type numbers via the covering space approach following $[\mathbf{J}]$ (compare [FW] and [W2]). The advantage of this approach is to be able to consider the empty fixed point classes which play an important role in relative Nielsen fixed point theory $([\mathbf{Z}])$. We show that these Nielsen type numbers enjoy the usual properties of the classical Nielsen number, in particular, the $G$-homotopy invariant property. In $\S 3$, we prove minimality theorems for fixed orbits (orbits of fixed points) and for fixed points. We also describe a procedure for computing the minimal number of fixed points in the $G$-homotopy class. Techniques employed here are those of the Wecken method used in relative Nielsen theory $([\mathbf{S}],[\mathbf{Z}])$ with a modification to the equivariant setting. Computations of the equivariant Nielsen numbers are carried out in $\S 4$ by introducing an equivariant analog of the Jiang condition. Under such condition we relate the equivariant Nielsen numbers to the equivariant fixed point indices in [K] (e.g. 4.12) and the ordinary Nielsen numbers $\left\{N\left(f^{n}\right)\right\}$. In $\S 5$, we indicate how the Nielsen theory of periodic points developed in $[\mathbf{J}]$, [HPY], [HY] relates to the equivariant Nielsen theory. We conclude in $\S 6$ with some final remarks.

The author would like to thank Jerzy Jezierski for pointing out an error in the definition of $N O_{G}\left(f^{H}\right)$ and the referee for a number of helpful suggestions.

2. Equivariant Nielsen type invariants. Let $W$ be a finite group and $Y$ be a connected compact $W$-ENR. let $\eta: \widetilde{Y} \rightarrow Y$ be the universal cover of $Y$ and $\operatorname{Cov}(\eta)$ be the group of deck transformations. We also identify $\operatorname{Cov}(\eta)$ with the fundamental group $\pi=\pi_{1}(Y)$ of $Y$. Consider the group

$$
\widetilde{W}=\{\tilde{\gamma} \in \operatorname{Homeo}(\tilde{Y}) \mid \eta \tilde{\gamma}=\gamma \eta \text { for some } \gamma \in W\}
$$

consisting of homeomorphisms of $\tilde{Y}$ covering the $W$-action on $Y$. Therefore we have a short exact sequence of groups

$$
1 \rightarrow \pi \hookrightarrow \widetilde{W} \stackrel{\eta}{\rightarrow} W \rightarrow 1 .
$$

Definition 2.1. Let $h: Y \rightarrow Y$ be a $W$-map. Two lifts $\tilde{h}$ and $\tilde{h}^{\prime}$ are said to be conjugate if there exists $\tilde{\gamma} \in \widetilde{W}$ such that $\tilde{h}^{\prime}=\tilde{\gamma} \tilde{h} \tilde{\gamma}^{-1}$ Denote by $[\tilde{h}]=\left\{\tilde{\gamma} \tilde{h} \tilde{\gamma}^{-1} \mid \tilde{\gamma} \in \widetilde{W}\right\}$ the conjugacy class of $\tilde{h}$.

Proposition 2.2. Let $h: Y \rightarrow Y$ be a $W$-map and $\tilde{h}, \tilde{h}^{\prime}$ be lifts of $h$. 
(1) If $[\tilde{h}]=\left[\tilde{h}^{\prime}\right]$ then $W(\eta$ Fix $\tilde{h})=W\left(\eta\right.$ Fix $\left.\tilde{h}^{\prime}\right)$.

(2) If $[\tilde{h}] \neq\left[\tilde{h}^{\prime}\right]$ then $W(\eta$ Fix $\tilde{h}) \cap W\left(\eta\right.$ Fix $\left.\tilde{h}^{\prime}\right)=\varnothing$.

Proof. (1) Suppose that $\tilde{h}^{\prime}=\tilde{\gamma} \tilde{h} \tilde{\gamma}^{-1}$ for some $\tilde{\gamma} \in \widetilde{W}$. Let $x \in$ $\eta$ Fix $\tilde{h}$ and $\tilde{x} \in \operatorname{Fix} \tilde{h} \cap \eta^{-1}(x)$. Then $\tilde{h}^{\prime}(\tilde{\gamma} \tilde{x})=\tilde{\gamma} \tilde{h} \tilde{\gamma}^{-1}(\tilde{\gamma} \tilde{x})=\tilde{\gamma} \tilde{h} \tilde{x}=$ $\tilde{\gamma} \tilde{x} \Rightarrow \tilde{\gamma} \tilde{x} \in \operatorname{Fix} \tilde{h}^{\prime}$. Thus, $\eta \tilde{\gamma} \tilde{x}=\gamma \eta \tilde{x}=\gamma x \in \eta$ Fix $\tilde{h}^{\prime}$ and hence $\gamma \eta$ Fix $\tilde{h} \subset \eta$ Fix $\tilde{h}^{\prime}$.

Conversely, let $y \in \eta \operatorname{Fix} \tilde{h}^{\prime}$ and $\tilde{y} \in \operatorname{Fix} \tilde{h}^{\prime} \cap \eta^{-1}(y)$. Then $\tilde{\gamma}^{-1} \tilde{y}=$ $\tilde{\gamma}^{-1} \tilde{h}^{\prime} \tilde{y}=\tilde{\gamma}^{-1}\left(\tilde{\gamma} \tilde{h} \tilde{\gamma}^{-1}\right) \tilde{y}=\tilde{h} \tilde{\gamma}^{-1} \tilde{y}$. Thus, $\tilde{\gamma}^{-1} \tilde{y} \in$ Fix $\tilde{h}$ and $\gamma^{-1} y=$ $\gamma^{-1} \eta \tilde{y}=\eta \tilde{\gamma}^{-1} \tilde{y} \in \eta$ Fix $\tilde{h}$. Hence $y \in \gamma \eta$ Fix $\tilde{h}$. The assertion follows from the fact that $\gamma \eta$ Fix $\tilde{h}=\eta$ Fix $\tilde{h}^{\prime}$.

(2) Suppose that $x_{0} \in W(\eta$ Fix $\tilde{h}) \cap W\left(\eta\right.$ Fix $\left.\tilde{h}^{\prime}\right)$. Then there exist $\gamma_{1}, \gamma_{2} \in W, \tilde{x}_{1} \in$ Fix $\tilde{h}$ and $\tilde{x}_{2} \in$ Fix $\tilde{h}^{\prime}$ such that $\gamma_{1} \eta \tilde{x}_{1}=x_{0}=$ $\gamma_{2} \eta \tilde{x}_{2}$. Thus there exist $\tilde{\gamma}_{1}, \tilde{\gamma}_{2} \in \widetilde{W}$ with $\eta \tilde{\gamma}_{1} \tilde{x}_{1}=\eta \tilde{\gamma}_{2} \tilde{x}_{2}$ and hence $\tilde{\gamma}_{2} \tilde{x}_{2}=\alpha \tilde{\gamma}_{1} \tilde{x}_{1}$ for some $\alpha \in \pi$. Let $\tilde{\gamma}=\tilde{\gamma}_{2}^{-1} \alpha \tilde{\gamma}_{1}$. We have $\tilde{x}_{2}=$ $\tilde{\gamma} \tilde{x}_{1}, \tilde{\gamma} \tilde{h} \tilde{\gamma}^{-1}\left(\tilde{x}_{2}\right)=\tilde{\gamma} \tilde{h} \tilde{\gamma}^{-1}\left(\tilde{\gamma} \tilde{x}_{1}\right)=\tilde{\gamma} \tilde{h} \tilde{x}_{1}=\tilde{\gamma} \tilde{x}_{1}=\tilde{x}_{2}$ and $\tilde{h}^{\prime}\left(x_{2}\right)=\tilde{x}_{2}$. By uniqueness of lifts, $\tilde{h}^{\prime}=\tilde{\gamma} \tilde{h} \tilde{\gamma}^{-1}$ and hence $[\tilde{h}]=\left[\tilde{h}^{\prime}\right]$.

Definition 2.3. Let $h: Y \rightarrow Y$ be a $W$-map and $\tilde{h}$ be a lift of $h$. The $W$-subset $W(\eta$ Fix $\tilde{h})$ is called the $W$-fixed point class determined by the conjugacy class $[\tilde{h}]$ (or simply $W$-fpc).

Proposition 2.4. Let $x, y \in$ Fix $h \neq \varnothing$. Then $x$ and $y$ belong to the same $W$-fpc if, and only if,

(1) $y=\sigma x$ for some $\sigma \in W$, or

(2) there exists a path $\alpha:[0,1] \rightarrow Y$ such that $\alpha(0)=x, \alpha(1)=$ $\sigma^{\prime} y$ for some $\sigma^{\prime} \in W$ and $\alpha \sim h \circ \alpha$ (rel endpoints).

Hence, there are finitely many non-empty $W$-fpcs.

Proof. We proceed as in [J, I.1.10 and 1.12].

Since each $W$-fpc $N$ of $h$ is open and closed in Fix $h$, we can assign the usual fixed point index $I(h, N)$ [D2] which is defined to be zero if $N$ is empty. If $I(h, N) \neq 0$ then $N$ is called an essential $W$-fpc of $h$.

Let $G$ be a compact Lie group and $X$ be a compact $G$-ENR. Let $\mathscr{F}=\{(H) \in \operatorname{Iso}(X)|| W H \mid<\infty\}$ where $W H=N H / H$ is the Weyl group and Iso $(X)$ is the set of isotropy types of $X$. We assume (for simplicity) that $X^{H}=\{x \in X \mid h x=x, \forall h \in H\}$ is connected for each $(H) \in \mathscr{F}$. For any $G$-map $f: X \rightarrow X$ and for each $(H) \in \mathscr{F}$, 
we have the notion of (essential) $W H$-fpcs by setting $W=W H$, $h=f^{H}$ and $Y=X^{H}$.

Let $f: X \rightarrow X$ be a $G$-map. For any $(H) \in \mathscr{F}$, an $(H)$-fpc of $f^{(H)}: X^{(H)} \rightarrow X^{(H)}$ is of the form $G N$ where $N$ is a $W H$-fpc of $f^{H}$ determined by some $\left[\tilde{f}^{H}\right]$ and $X^{(H)}=\{x \in X \mid(G x) \geq(H)\}$. Denote by $\mathrm{FPC}_{(H)}(f)$ the set of $(H)$-fpcs of $f^{(H)}$. We also let $X_{H}=\{x \in$ $\left.X \mid G_{X}=H\right\}$ and $f_{H}=f \mid X_{H}: X_{H} \rightarrow X^{H}$.

Let $(K) \in \mathscr{F}$ and $N^{\prime}$ be a $W K$-fpc so that $N^{\prime}=W K\left(p_{K}\right.$ Fix $\left.\tilde{f}^{K}\right)$ for some lift $\tilde{f}^{K}$ of $f^{K}$. Suppose that $H \leq K$ and $(H) \in \mathscr{F}$. There is a unique lift $\tilde{f}^{H}$ of $f^{H}$ such that $p_{K}$ Fix $\tilde{f}^{K} \subset p_{H}$ Fix $\tilde{f}^{H}$. Thus $G N^{\prime} \subset G N$ where $N=W H\left(p_{H}\right.$ Fix $\left.\tilde{f}^{H}\right)$. There is a (contravariant) function $\tau_{(H) \leq(K)}: \operatorname{FPC}_{(K)}(f) \rightarrow \operatorname{FPC}_{(H)}(f)$ for $(H) \leq(K) \in \mathscr{F}$. In this case, $\tau_{(H) \leq(K)}\left(G N^{\prime}\right)=G N$.

Definiton 2.5. Let $f: X \rightarrow X$ be a $G$-map. For each $(H) \in \mathscr{F}$, define

$$
\begin{aligned}
& N_{W H}\left(f^{H}\right)=\#\{\text { essential } W H \text {-fpcs }\}, \\
& N O_{G}\left(f_{H}\right)=\#\left\{\text { essential } W H \text {-fpc } N \mid \tau_{(H) \leq(K)}\left(G N^{\prime}\right) \neq G N\right. \\
& \text { for all essential } \left.W K \text {-fpc } N^{\prime},(H)<(K)\right\}, \\
& N_{G}\left(f_{H}\right)=|W H| \cdot \#\left\{\text { essential } W H \text {-fpc } N \mid \tau_{(H) \leq(K)}\left(G N^{\prime}\right) \neq G N\right. \\
& \text { for all } \left.W K \text {-fpc } N^{\prime},(H)<(K)\right\}, \\
& N O_{G}\left(f^{H}\right)=\min \left\{\# \mathscr{C} \mid \mathscr{C} \subset \bigcup_{(H) \leq(K)} \operatorname{FPC}_{(K)}(f)\right. \text {, for any essential } \\
& W L \text {-fpc } N^{\prime}, \exists \text { a } W K \text {-fpc } N \in \mathscr{C} \text { such that } \\
& \left.\tau_{(L) \leq(K)}(G N)=G N^{\prime}\right\} \\
& N_{G}\left(f^{H}\right)=\sum_{(H) \leq(K) \in \mathscr{F}} N_{G}\left(f_{K}\right) .
\end{aligned}
$$

We now show the $G$-homotopy invariance and lower bound properties.

Proposition 2.6 ( G-Homotopy Invariance). Given any G-homotopy $\varphi \sim_{G} f$,

(1) $N_{W H}\left(f^{H}\right)=N_{W H}\left(\varphi^{H}\right)$,

(2) $N O_{G}\left(f_{H}\right)=N O_{G}\left(\varphi_{H}\right)$,

(3) $N_{G}\left(f_{H}\right)=N_{G}\left(\varphi_{H}\right)$,

(4) $N O_{G}\left(f^{H}\right)=N O_{G}\left(\varphi^{H}\right)$, and

(5) $N_{G}\left(f^{H}\right)=N_{G}\left(\varphi^{H}\right)$

for every $(H) \in \mathscr{F}$. 
Proof. Let $F: X \times[0,1] \rightarrow X$ be a $G$-homotopy with $F_{0}=f$ and $F_{1}=\varphi$ and let $(H) \in \mathscr{F}$. Note that $F_{t}^{H}: X^{H} \rightarrow X^{H}$ is a $W H$-map for $t \in[0,1]$. For $i=0,1$, let $N_{i}$ be a nonempty $W H$-fpc of $F_{i}^{H}$. We say that $N_{0}$ and $N_{1}$ are $F^{H}$-related if there exists $x_{0} \in N_{0}$, $x_{1} \in N_{1}$ and a path $\left\{x_{t}\right\}_{t \in[0,1]}$ in $X^{H}$ such that $\left\{F_{t}^{H}\left(x_{t}\right)\right\} \sim\left\{x_{t}\right\}$ (rel endpoints). Let $\widehat{F}: X \times[0,1] \rightarrow[0,1]$ be defined by $\widehat{F}(x, t)=$ $(F(x, t), t)$ and $\widehat{F}^{H}=\widehat{F} \mid X^{H} \times[0,1]$.

It follows that $N_{0}$ and $N_{1}$ belong to the same (nonempty) $W H$-fpc of $\widehat{F}^{H}$ if they are $F^{H}$-related, in which case $I\left(F_{0}^{H}, N_{0}\right)=I\left(F_{1}^{H}, N_{1}\right)$ (see [J, I.3.10]). If $N_{0}$ is not $F^{H}$-related to any nonempty $W H$-fpc of $F_{1}^{H}$, then $I\left(F_{0}^{H}, N_{0}\right)=0$. Hence there is a one-to-one correspondence between the essential $W H$-fpcs of $F_{0}^{H}$ and those of $F_{1}^{H}$. Thus, (1) holds.

Let $N$ be an essential $W H$-fpc of $f^{H}=F_{0}^{H}$ and $M$ be the corresponding essential $W H$-fpc of $\varphi^{H}=F_{1}^{H}$. Suppose that for some $(K) \in \mathscr{F},(H)<(K)$ and $\tau_{(H) \leq(K)}\left(G M^{\prime}\right)=G M$, i.e., there exists a lift $\tilde{\varphi}^{K}$ of $\varphi^{K}$ such that $M^{\prime}=W K\left(p_{K}\right.$ Fix $\left.\tilde{\varphi}^{K}\right)$. There exists a homotopy $\widetilde{F}^{K}: \widetilde{X}^{K} \times[0,1] \rightarrow \widetilde{X}^{K}$ covering $F^{K}$ with $\widetilde{F}_{1}^{K}=\tilde{\varphi}^{K}$. Now $\tilde{f}^{K}=\widetilde{F}_{0}^{K}$ is a lift of $f^{K}$ and $N^{\prime}=W K\left(p_{K}\right.$ Fix $\left.\tilde{f}^{K}\right)$ is a $W K$-fpc of $f^{K}$. It follows that $\tau_{(H) \leq(K)}\left(G N^{\prime}\right)=G N$ and (3) follows.

To prove (2), we need to show that if $M^{\prime}$ is essential then so is $N^{\prime}$. This is equivalent to stating that $M^{\prime}$ and $N^{\prime}$ are $F^{K}$-related. Assertion (4) follows from the fact that essential fixed point classes do not disappear under homotopy and (5) follows immediately from (3).

Definition 2.7. Let $f: X \rightarrow X$ be a $G$-map. For each $(H) \in$ $\operatorname{Iso}(X)$, define

$$
\begin{aligned}
M O_{G}\left(f^{H}\right) & =\min \left\{\# \text { fixed orbits of } \varphi^{H} \mid \varphi \sim_{G} f\right\}, \\
M_{G}\left(f_{H}\right) & =\min \left\{\#\left(\operatorname{Fix} \varphi_{H}\right) \mid \varphi \sim_{G} f\right\} .
\end{aligned}
$$

Proposition 2.8 (Lower bound). For each $(H) \in \mathscr{F}$,

(1) $M O_{g}\left(f^{H}\right) \geq N O_{G}\left(f^{H}\right)$ [fixed orbits in $X^{H}$ ].

(2) $M_{G}\left(f_{H}\right) \geq N_{G}\left(f_{H}\right)$ [fixed points in $X_{H}$ ].

Proof. (1) Let $(H) \in \mathscr{F}$. Choose an admissible ordering $\left(H_{1}\right), \ldots$, $\left(H_{m}\right)$ on $\{(K) \mid(K) \geq(H)\}$ with the associated filtration of $G$-subspaces $X_{1} \subset \cdots \subset X_{m}=X^{(H)}$. If $m=1$, then $N O_{G}\left(f^{H}\right)=N O_{G}\left(f_{H}\right)=$ $N_{W H}\left(f^{H}\right) \leq M O_{G}\left(f^{H}\right)$. Assume that $M O_{G}\left(f^{H_{i}}\right) \geq N O_{G}\left(f^{H_{i}}\right)$ for all $i, 1 \leq i<m$. Suppose that $\varphi \sim_{G} f$ such that $\varphi^{H}$ has $M O_{G}\left(f^{H}\right)$ 
fixed orbits in $X^{H}$. Let $\mathscr{C} \subset \bigcup_{(H) \leq(K)} \operatorname{FPC}_{(K)}(f)$ such that $N O_{G}\left(\varphi^{H}\right)$ $=N O_{G}\left(f^{H}\right)=\# \mathscr{C}$. If $G N^{\prime} \in \mathscr{C}$, then it corresponds to some essential $G N^{\prime}$. By the homotopy invariance of $N_{W H}\left(f^{H}\right), \varphi^{H}$ must contain at least $\# \mathscr{C}$ fixed orbits.

(2) If $N$ is an essential $W H$-fpc such that $G N \cap X_{(K)} \neq \varnothing$ for some $(H)<(K) \in \mathscr{F}$, then $\tau_{(H) \leq(K)}\left(G N^{\prime}\right)=G N$ for some $W K$-fpc $N^{\prime}$ (not necessary essential). Thus, for any $\varphi \sim_{G} f, \varphi_{(H)}$ must have at least $N_{G}\left(f_{H}\right) /|W H|$ fixed orbits in $X_{(H)}=X^{(H)}-\bigsqcup_{(H)<(K)} X_{(K)}$. Hence in $X_{H}$, we have $M_{G}\left(f_{H}\right) \geq N_{G}\left(f_{H}\right)$.

In classical Nielsen fixed point theory, the ordinary Nielsen number possesses the commutativity and homotopy type invariant properties. The following are the corresponding equivariant analogs of these two properties. The proofs are straightforward so we leave them to the reader.

Proposition 2.9. Let $X$ and $Y$ be compact $G$-ENRs and $\mathscr{H}=$ $\{(H) \in \operatorname{Iso}(X) \cup \operatorname{Iso}(Y)|| W H \mid<\infty\}$. Suppose that $X^{H}$ and $Y^{H}$ are connected for every $(H) \in \mathscr{H}$.

(1) (G-commutativity) Let $f: X \rightarrow Y$ and $g: Y \rightarrow X$ be G-maps. Then

$$
\begin{aligned}
N O_{G}\left((g \circ f)^{H}\right) & =N O_{G}\left((f \circ g)^{H}\right), \\
N_{G}\left((g \circ f)^{H}\right) & =N_{G}\left((f \circ g)^{H}\right)
\end{aligned}
$$

for all $(H) \in \mathscr{H}$.

(2) (G-Homotopy Type Invariance) Given the following commutative diagram

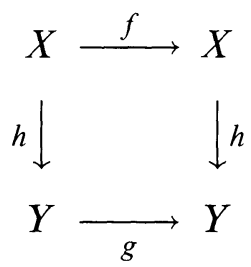

where all maps are G-maps and $h$ is a G-homotopy equivalence with inverse $k$. Then

$$
\begin{aligned}
N O_{G}\left(f^{H}\right) & =N O_{G}\left(g^{H}\right), \\
N_{G}\left(f^{H}\right) & =N_{G}\left(g^{H}\right)
\end{aligned}
$$

for all $(H) \in \mathscr{H}$.

3. Minimality theorems. For most of the results in this section, we make the following Standard Hypotheses on $G$ and $X$ : 
STANDARD HYPOTHESES. Let $G$ be a compact Lie group and $X$ be a compact smooth $G$-manifold. For each $(H) \in \mathscr{F}$, we assume that $X^{H}$ is connected, $\operatorname{dim} X^{H} \geq 3$ and $\operatorname{dim} X^{H}-\operatorname{dim}\left(X^{H}-X_{H}\right) \geq 2$.

Note that $\mathscr{F}=\operatorname{Iso}(X)$ when $G$ is finite.

In classical Nielsen fixed point theory, the Wecken method for coalescing fixed points in the same class is crucial in proving the minimality theorem. The following is the equivariant analog.

Lemma 3.1. Assume the Standard Hypotheses. Suppose that $f: X \rightarrow$ $X$ is a G-map, $\mathscr{O}_{1}$ and $\mathscr{O}_{2}$ are two distinct isolated $W H$-fixed orbits belonging to the same $W H$-fpc of $f^{H}$, for some $(H) \in \mathscr{F}$. Furthermore, we assume that $G \mathscr{O}_{1} \subset X_{(H)}, G \mathscr{O}_{2} \subset X_{(K)}$ for some $(K) \in \mathscr{F}$ with $(H) \leq(K)$. Then there exists a G-homotopy $\left\{f_{t}\right\}$ relative to $X^{>(H)}=\left\{x \in X \mid\left(G_{x}\right)>(H)\right\}$ such that $f_{0}=f$ and Fix $f_{1}=$ Fix $f_{0}-G \mathscr{O}_{1}$.

Proof. Suppose that $(H)<(K)$. There exist $x_{1} \in \mathscr{O}_{1}, x_{2} \in \mathscr{O}_{2}$ and a path $\sigma:[0,1] \rightarrow X^{H}$ such that $\sigma(0)=x_{1}, \sigma(1)=x_{2}$ and $\sigma \sim f^{H} \circ \sigma$ (rel endpoints). Since $x_{1} \in X_{H}$ and $x_{2} \in X^{H}-X_{H}$ and $\operatorname{dim} X^{H}-\operatorname{dim}\left(X^{H}-X_{H}\right) \geq 2$, we may assume that $\sigma$ can be chosen so that $\sigma([0,1)) \subset X_{H}$. We coalesce $x_{1}$ and $x_{2}$ along $\sigma$ as in [W1, 1.1] (see also [S, 6.1]). Taking the $G$-translates of $N(\sigma)$, we move $G \mathscr{O}_{1}$ to $G \mathscr{O}_{2}$ along the paths $G \sigma$ in $G N(\sigma)$. For the case $(H)=(K)$, it follows from [W2, 5.4] since $X_{H}$ is a free $W H$-space.

THeOREM 3.2. Assume the Standard Hypotheses. Given a G-map $f: X \rightarrow X$, there exists a G-map $h \sim_{G} f$ such that

$$
N O_{G}\left(h^{H}\right)=N O_{G}\left(f^{H}\right)=M O_{G}\left(f^{H}\right)
$$

for all $(H) \in \mathscr{F}$.

Proof. First we can $G$-homotope $f$ to $f^{\prime}$ with only a finite number of fixed orbits. If $(H) \in \operatorname{Iso}(X)$ with $|W H|=\infty$, then we can remove the fixed orbits in $X_{(H)}$ [FW, 2.3]. Thus we may assume that Fix $f^{\prime} \subset \bigcup_{(K) \in \mathscr{F}} X_{(K)}$. Furthermore, if $N$ is a fixed orbit in $X_{H}$ for some $(H) \in \mathscr{F}$ with $I\left(f^{H}, N\right)=0$ then we can remove $N$ and hence the entire $G$-orbit $G N$.

Fix an $(H) \in \mathscr{F}$ and choose an admissible ordering on $\left\{\left(H_{i}\right) \in\right.$ $\left.\mathscr{F} \mid(H) \leq\left(H_{i}\right)\right\}$ with $X_{1} \subset \cdots \subset X_{m}=X^{(H)}$. If $m=1$, then the assertion follows by removing the inessential fixed orbits and by applying 3.1 to those orbits of the same class. Suppose that $N O_{G}\left(f^{H_{i}}\right)=$ 
$M O_{G}\left(f^{H_{i}}\right)$ for $1 \leq i<m$. Let $\varphi \sim_{G} f$ such that $M O_{G}\left(\varphi^{H_{m-1}}\right)=$ $N O_{G}\left(\varphi^{H_{m-1}}\right)$ and $\varphi_{H}=\varphi_{H_{m}}$ has only isolated fixed orbits of nonzero index in $X_{H}$. Apply 3.1 to these fixed orbits in $X_{H}$. We arrive at a $G$-map $h \sim_{G} f$ such that $N O_{G}\left(h^{H_{\imath}}\right)=M O_{G}\left(f^{H_{\imath}}\right)$ for $1 \leq i<m$ and $h_{H}$ has exactly $N O_{G}\left(f_{H}\right)$ fixed orbits that cannot be coalesced with any of the essential fixed orbits in $X^{H}-X_{H}$. Hence $N O_{G}\left(h^{H}\right)$ is mininal.

Theorem 3.3. Assume the Standard Hypotheses and let $f: X \rightarrow X$ be a $G$-map. For each $(H) \in \operatorname{Iso}(X)$, there exists a G-map $h \sim_{G} f$ such that

$$
M_{G}\left(f_{H}\right)=\#\left(\operatorname{Fix} h_{H}\right)= \begin{cases}N_{G}\left(f_{H}\right) & \text { if }(H) \in \mathscr{F} \\ 0 & \text { otherwise }\end{cases}
$$

Proof. If $|W H|=\infty$ then the assertion is obvious. Suppose that $(H) \in \mathscr{F}$. We can $G$-deform $f$ to a $G$-map $\varphi$ such that $\varphi$ has only isolated fixed orbits. Proceed as in the proof of 3.2. We then arrive at a $G$-map $\psi \sim_{G} f$ so that $\psi_{H}$ has exactly $N O_{G}\left(f_{H}\right)$ fixed orbits in $X_{H}$. We can further unite those essential $W H$-fpcs to the inessential $W K$-fpcs that they correspond to for some $(H)>(H)$ by applying 3.1. (It should be noted that if the inessential $W K$-fpc is empty, we need to "create" a fixed orbit of index zero [W1, 1.1] before applying 3.1.) It then follows that $\#\left(\operatorname{Fix} h_{H}\right)=N_{G}\left(f_{H}\right)$.

REMARK 3.4. In general, we may not be able to find a $G$-homotopy $h \sim_{G} f$ such that $M_{G}\left(f_{H}\right)=N_{G}\left(f_{H}\right)$ for all $(H) \in \mathscr{F}$ simultaneously. For example, take $G=\mathbf{Z}_{2}$ acting on $X=S^{4}$ as an involution so that $X^{G}=S^{3}$. Let $f: X \rightarrow X$ be the identity map. It is easy to see that $M_{G}\left(f_{G}\right)=0=M_{G}\left(f_{(1)}\right)$ but the minimal number of fixed points in the $G$-homotopy class of the identity is equal to 1 .

We now describe a procedure of computing the minimal number of fixed points in the $G$-homotopy class of a $G$-map when $G$ is a finite group.

Let $G$ be a finite group and $X$ be a compact $G$-ENR. Given a $G$-map $f: X \rightarrow X$, for each $(H) \in \operatorname{Iso}(X)$, let

$$
\begin{aligned}
\mathscr{N}_{f}(H)=\left\{G N \mid I\left(f^{H}, N\right)\right. & \neq 0 \text { and for all }(K)>(H), \\
& \left.I\left(f^{K}, N^{\prime}\right) \neq 0 \Rightarrow \tau_{(H) \leq(K)}\left(G N^{\prime}\right) \neq G N\right\}
\end{aligned}
$$

and

$$
\mathscr{P}_{f}(H)=\left\{(K)>(H) \mid \exists G N \in \mathscr{N}_{f}(H), \tau_{(H) \leq(K)}\left(G N^{\prime}\right)=G N\right.
$$

for some $N^{\prime}$ with $\left.I\left(f^{K}, N^{\prime}\right)=0\right\}$. 
For any $\left(K_{1}\right),\left(K_{2}\right) \in \mathscr{P}_{f}(H)$, we write $\left(K_{1}\right) \leq_{f^{H}}\left(K_{2}\right)$ if

(1) $\left(K_{1}\right) \leq\left(K_{2}\right)$ and

(2) there exists a $G N \in \mathscr{N}_{f}(H)$ such that $\tau_{(H) \leq\left(K_{1}\right)}\left(G N_{1}\right)=G N=$ $\tau_{(H) \leq\left(K_{2}\right)}\left(G N_{2}\right)$ for some $\left(K_{1}\right)$-fpc $G N_{1}$ and $\left(K_{2}\right)$-fpc $G N_{2}$.

Let

$$
\mathscr{M}_{f}(H)=\left\{(K) \in \mathscr{P}_{f}(H) \mid(K) \text { maximal with respect to } \leq_{f^{H}}\right\} .
$$

For any $\left(K_{1}\right),\left(K_{2}\right) \in \mathscr{M}_{f}(H)$, we write $\left(K_{1}\right) \preceq\left(K_{2}\right)$ if $\left|K_{1}\right| \leq\left|K_{2}\right|$.

Definition 3.5. For any $\left(K_{1}\right) \preceq\left(K_{2}\right) \in \mathscr{M}_{f}(H)$, let

$$
\begin{aligned}
& \zeta_{(H)}\left(\left(K_{1}\right),\left(K_{2}\right)\right) \\
&=\#\left\{G N \in \mathcal{N}_{f}(H) \mid \exists G N_{1} \text { such that } \tau_{(H) \leq\left(K_{1}\right)}\left(G N_{1}\right)=G N\right. \\
&\text { and } \left.\tau_{(H) \leq\left(K_{2}\right)}\left(G N_{2}\right) \neq G N \text { for all } G N_{2}\right\} .
\end{aligned}
$$

Note that

$$
\sum_{(K) \preceq(L)} \zeta_{(H)}((K),(L))
$$

is the number of $N \in \mathscr{N}_{f}(H)$ that contain a $K$-fpc but not an $L$-fpc for any $(L) \succ(K)$.

Definition 3.6. Let $(H) \in \operatorname{Iso}(X)$. The minimal number of fixed points in $X^{(H)}$ in the $G$-homotopy class of a $G$-map $f: X \rightarrow X$ is denoted by

$$
m_{G}\left(f^{(H)}\right)=\min \left\{\#\left(\operatorname{Fix} h^{(H)}\right) \mid h \sim_{G} f\right\} .
$$

In the proof of 3.2 , we showed that $f$ is $G$-homotopic to a $G$ map $h$ with exactly $N O_{G}\left(f^{H}\right)$ fixed orbits in $X^{H}$ (or in $X^{(H)}$ ) in which $h$ has at most $N O_{G}\left(f_{H}\right)$ fixed orbits in $X_{H}$. In order to minimize the number of fixed points we should further coalesce those $(H)$-fpcs among the $N O_{G}\left(f_{H}\right)$ ones, to the inessential $(K)$-fpcs that they contain for $(K) \geq(H)$ as in 3.3. For each $(H)$, there are exactly $\left(N O_{G}\left(f_{H}\right)-N_{G}\left(f_{H}\right) /|W H|\right) W H$-fpcs that can be further united to some lower strata. These fixed point classes can be coalesced with some inessential $(K)$-fpc where $(K) \in \mathscr{M}_{f}(H)$. Since it is possible that some $G N \in \mathscr{N}_{f}(H)$ can contain a $\left(K_{1}\right)$ - and a $\left(K_{2}\right)$-fpc for distinct $\left(K_{1}\right),\left(K_{2}\right) \in \mathscr{M}_{f}(H)$, we use the partial ordering $\preceq$ on $\mathscr{M}_{f}(H)$ to decide where $G N$ should be moved to. Thus for each $(K) \in \mathscr{M}_{f}(H)$, we should move to $X_{(K)}$ those fixed point classes that contain a $(K)$ fpc but not an $(L)$-fpc for any $(L) \succ(K)$. This procedure can be summarized by the following 
Theorem 3.7. Assume the Standard Hypotheses with $G$ finite. Given any G-map $f: X \rightarrow X$ and $(H) \in \operatorname{Iso}(X)$,

$$
N O_{G}\left(f_{H}\right)=\frac{N_{G}\left(f_{H}\right)}{|W H|}+\sum_{(K) \in \mathscr{M}_{f}(H)} \sum_{(K) \preceq(L)} \zeta_{(H)}((K),(L))
$$

and

$$
\begin{aligned}
m_{G}\left(f^{(H)}\right)=\sum_{(H) \leq(K)} & {\left[N_{G}\left(f_{K}\right) \cdot[G: N K]\right.} \\
& \left.+\sum_{\left(K^{\prime}\right) \in \mathscr{M}_{f}(K)} \sum_{\left(K^{\prime}\right) \preceq(L)} \zeta_{(K)}\left(\left(K^{\prime}\right),(L)\right) \cdot\left[G: K^{\prime}\right]\right] .
\end{aligned}
$$

REMARK 3.8. A similar but simpler formula for $m_{G}\left(f^{(H)}\right)$ was also obtained in [W1, 2.2] for the special case $f=1_{X}$. From 3.7, we obtain the following inequality

$$
m_{G}\left(f^{(H)}\right) \geq \sum_{(H) \leq(K)} N_{G}\left(f_{K}\right) \cdot[G: N K] \geq \sum_{(H) \leq(K)} N O_{G}\left(f_{K}\right) \cdot[G: K] .
$$

EXAMPLE 3.9. Let $X=S^{1} \times S^{1} \times S^{1} \times S^{1} \times S^{1} \times S^{2}$ and $G=\mathbf{Z}_{6}=$ $\langle\alpha\rangle \times\langle\beta\rangle$ where $\mathbf{Z}_{2}=\langle\alpha\rangle, \mathbf{Z}_{3}=\langle\beta\rangle$. Let $G$ act on $X$ via $\alpha \cdot\left(e^{i \theta_{1}}, \ldots, e^{i \theta_{5}},(x, y, z)\right)=\left(e^{i \theta_{2}}, e^{i \theta_{1}}, e^{i \theta_{3}}, e^{i \theta_{4}}, e^{i \theta_{5}},(x, y,-z)\right)$, $\beta \cdot\left(e^{i \theta_{1}}, \ldots, e^{i \theta_{5}},(x, y, z)\right)=\left(e^{i \theta_{1}}, e^{i \theta_{2}}, e^{i \theta_{5}}, e^{i \theta_{3}}, e^{i \theta_{4}},(x, y, z)\right)$.

Then

$$
\begin{aligned}
X^{\langle\alpha\rangle} & =\left\{\left(e^{i \theta}, e^{i \theta}, e^{i \theta_{3}}, e^{i \theta_{4}}, e^{i \theta_{5}},(x, y, 0)\right)\right\} \approx T^{5}, \\
X^{\langle\beta\rangle} & =\left\{\left(e^{i \theta_{1}}, e^{i \theta_{2}}, e^{i \theta}, e^{i \theta}, e^{i \theta},(x, y, z)\right)\right\} \approx T^{3} \times S^{2}, \\
X^{G} & =\left\{\left(e^{i a}, e^{i a}, e^{i b}, e^{i b}, e^{i b},(x, y, 0)\right)\right\} \approx T^{3} .
\end{aligned}
$$

Let $f: X \rightarrow X$ be the $G$-map defined by

$$
\begin{aligned}
& f\left(e^{i \theta_{1}}, \ldots, e^{i \theta_{5}},(x, y, z)\right) \\
& \quad=\left(e^{i 2 \theta_{2}}, e^{i 2 \theta_{1}}, e^{i 2 \theta_{3}}, e^{i 2 \theta_{4}}, e^{i 2 \theta_{5}},(x,-y,-z)\right) .
\end{aligned}
$$

Then $f$ has eight fixed points given by

$$
\text { Fix } f=\left\{\left(m, m^{2}, 1,1,1,(n, 0,0)\right) \mid m^{3}=1 ; n=1,-1\right\} \text {. }
$$

Furthermore,

$$
\text { Fix } f^{G}=\operatorname{Fix} f^{\langle\alpha\rangle}=\{(1,1,1,1,1, m) \mid m=1,-1\}
$$


and Fix $f^{\langle\beta\rangle}=\operatorname{Fix} f$. The ordinary Nielsen numbers are given by

$$
N(f)=3 ; \quad N\left(f^{\langle\alpha\rangle}\right)=2 ; \quad N\left(f^{\langle\beta\rangle}\right)=3 ; \quad N\left(f^{G}\right)=2 .
$$

Let us compute the minimal number of fixed orbits and of fixed points in the $G$-homotopy class of $f$.

First of all, Fix $f$ consists of three distinct essential (ordinary) fixed point classes of $f$ with $N_{G}\left(f_{G}\right)=N_{G}\left(f^{G}\right)=N\left(f^{G}\right)=2, N_{G}\left(f_{\langle\alpha\rangle}\right)=$ $0, N_{G}\left(f_{\langle\beta\rangle}\right)=4$ and $N_{G}\left(f_{(1)}\right)=0$.

Furthermore, $\mathscr{M}_{f}((1))=\{\langle\beta\rangle, G\}, \mathscr{M}_{f}(\langle\alpha\rangle)=\{G\}=\mathscr{M}_{f}(\langle\beta\rangle)$, $\mathscr{M}_{f}(G)=\varnothing$ and

$$
\begin{gathered}
N O_{G}\left(f_{G}\right)=2 ; \quad N O_{G}\left(f_{\langle\alpha\rangle}\right)=0 ; \quad N O_{G}\left(f_{\langle\beta\rangle}\right)=2 ; \\
N O_{G}\left(f_{(1)}\right)=0 \quad \text { and all } \zeta_{(H)}((K),(L))=0 .
\end{gathered}
$$

Hence, by 3.7 ,

$$
N O_{G}(f)=N O_{G}\left(f^{(1)}\right)=\sum_{(H)} \frac{N_{G}\left(f_{H}\right)}{|W H|}=2+0+2+0=4
$$

and

$$
m_{G}(f)=m_{G}\left(f^{(1)}\right)=2 \cdot 1+0 \cdot 1+4 \cdot 1+0 \cdot 1=6
$$

since $[G: N K]=1$ for all $(K) \in \operatorname{Iso}(X)$.

We conclude that $f$ is minimal in its $G$-homotopic class and $f$ has exactly four fixed orbits in which two lie in $X^{G}$ and the other two lie in $X_{\langle\beta\rangle}$. Equivalently, $f$ has two fixed points in $X^{G}$ and four fixed points in $X_{\langle\beta\rangle}$ which form two distinct $W\langle\beta\rangle \cong\langle\alpha\rangle$-orbits.

4. Computation. As we have seen in $\S 3$, the Nielsen type invariants $N O_{G}\left(f_{H}\right)$ and $N_{G}\left(f_{H}\right)$ are the basic ingredients in obtaining the minimality Theorems 3.2, 3.3 and 3.7. However they are in general, very difficult to compute. In this section, we approximate $N O_{G}\left(f_{H}\right)$ and $N_{G}\left(f_{H}\right)$ by $N_{W H}\left(f^{H}\right)$ which can be computed under certain conditions.

From Definition 2.6, for every $(H) \in \mathscr{F}$ we have

$$
N_{W H}\left(f^{H}\right) \geq N O_{G}\left(f_{H}\right)
$$

and

$$
N_{W H}\left(f^{H}\right) \geq N_{G}\left(f_{H}\right) /|W H| \text {. }
$$

Therefore,

$$
N O_{G}\left(f^{H}\right) \leq \sum_{(H) \leq(K) \in \mathscr{F}} N_{W K}\left(f^{K}\right)
$$


and

$$
N_{G}\left(f^{H}\right) \leq \sum_{(H) \leq(K) \in \mathscr{F}}|W K| \cdot N_{W K}\left(f^{K}\right) .
$$

Together with 3.2 and 3.3 , we obtain

Proposition 4.1. Let $G$ and $X$ be as in 3.1. For any G-map $f: X \rightarrow X$,

$$
M O_{G}\left(f^{H}\right) \leq \sum_{(H) \leq(K) \in \mathscr{F}} N_{W K}\left(f^{K}\right)
$$

and

$$
M_{G}\left(f_{H}\right) \leq|W H| \cdot N_{W H}\left(f^{H}\right) .
$$

For the minimal number of fixed points, we obtain from 3.7 the following

Proposition 4.2. Let $G$ and $X$ be as in 3.7. For any G-map $f: X \rightarrow X$ and $(H) \in \operatorname{Iso}(X)$,

$$
m_{G}\left(f^{(H)}\right) \geq \sum_{(H) \leq(K) \in \operatorname{Iso}(X)}[G: K] \cdot N_{W K}\left(f^{K}\right) .
$$

Let $W$ be a finite group and $Y$ be a connected compact $W$-ENR with universal cover $\widetilde{Y}$. Let $h: Y \rightarrow Y$ be a $W$-map. Fix a lift $\tilde{h}: \widetilde{Y} \rightarrow \widetilde{Y}$ of $h$. Recall from $\S 2$, there is a subgroup $\widetilde{W} \subset \operatorname{Homeo}(\widetilde{Y})$ covering $W$. For every $\tilde{\gamma} \in \widetilde{W}$, there exists a unique element $\varphi_{W}(\tilde{\gamma}) \in$ $\widetilde{W}$ such that $\varphi_{W}(\tilde{\gamma}) \tilde{h}=\tilde{h}_{\tilde{\gamma}}$. Thus we obtain a homomorphism $\varphi_{W}$ : $\widetilde{W} \rightarrow \widetilde{W}$. Note that any lift of $h$ is of the form $\alpha \tilde{h}$ for some $\alpha \in$ $\pi=\pi_{1}(Y)$.

Proposition 4.3. Two lifts $\alpha \tilde{h}$ and $\beta \tilde{h}$ are conjugate if, and only if, there exists $\tilde{\gamma} \in \widetilde{W}$ such that $\beta=\tilde{\gamma} \alpha \varphi_{W}(\tilde{\gamma})^{-1}$.

Proof. The lifts $\alpha \tilde{h}$ and $\beta \tilde{h}$ are conjugate iff $\beta \tilde{h}=\tilde{\gamma} \alpha \tilde{h} \tilde{\gamma}^{-1}$ for some $\tilde{\gamma} \in \widetilde{W}$

$$
\begin{aligned}
& \Leftrightarrow \beta \tilde{h} \tilde{\gamma}=\tilde{\gamma} \alpha \tilde{h} \\
& \Leftrightarrow \beta \varphi_{W}(\tilde{\gamma}) \tilde{h}=\tilde{\gamma} \alpha \tilde{h} \\
& \Leftrightarrow \beta \varphi_{W}(\tilde{\gamma})=\tilde{\gamma}_{\alpha} \\
& \Leftrightarrow \beta=\tilde{\gamma} \alpha \varphi_{W}(\tilde{\gamma})^{-1} .
\end{aligned}
$$


Definition 4.4. The group $\widetilde{W}$ acts on $\pi$ via $\alpha \mapsto \tilde{\gamma} \alpha \varphi_{W}(\tilde{\gamma})^{-1}$. Denote by $R\left(\varphi_{W}, \pi\right)$ the set of orbits of this action, called the set of $W$ Reidemeister classes of $\varphi_{W}$ on $\pi$. When restricted to $\pi, \varphi_{W} \mid \pi: \pi \rightarrow$ $\pi$ and hence the Reidemeister action reduces to the ordinary one studied in $[\mathbf{F H}]$.

REMARK 4.5. It follows from 2.2 that $W(\eta$ Fix $\alpha \tilde{h})=W(\eta$ Fix $\beta \tilde{h})$ iff $\beta=\tilde{\gamma} \alpha \varphi_{W}(\tilde{\gamma})^{-1}$ for some $\tilde{\gamma} \in \widetilde{W}$. Hence there is a one-to-one correspondence between the set of $W$-fpcs determined by $[\tilde{h}]$ and $R\left(\varphi_{W}, \pi\right)$.

DEFINITION 4.6. Let

$J_{W}(Y)=\{\alpha \in \pi \mid$ there exists a $W$-cyclic homotopy

$$
\left.1_{Y} \sim_{W} 1_{Y} \text { which can be lifted to } 1_{\widetilde{Y}} \sim \alpha\right\} .
$$

It is straightforward to verify the following

Proposition 4.7. $J_{W}(Y)$ is a subgroup of $J(Y)$, the Jiang subgroup of $Y$. We call $J_{W}(Y)$ the $W$-Jiang subgroup of $Y$.

Definition 4.8. Let $G$ be a compact Lie group and $X$ be a compact $G$-ENR. For every $(H) \in \mathscr{F}$, we assume that $X^{H}$ is connected. We say that $X$ is a $G$-Jiang space if for every $(H) \in \mathscr{F}$, $J_{W H}\left(X^{H}\right)=\pi_{1}\left(X^{H}\right)$.

In particular, if $X$ satisfies the $G$-Jiang condition then $X^{H}$ is a Jiang space for all $(H) \in \mathscr{F}$.

Proposition 4.9. Let $G$ be a finite group acting freely on a compact connected G-ENR $X$. If $X / G$ is a Jiang space then $X$ is a G-Jiang space.

Proof. Note that the Jiang subgroup of $X / G$ is given by

$$
\begin{array}{r}
J(X / G)=\left\{\bar{\alpha} \in \pi_{1}(X / G) \mid \exists \text { cyclic homotopy } 1_{X / G} \sim 1_{X / G}\right. \\
\text { which can be lifted to } \left.1_{\widetilde{X} / G} \sim \bar{\alpha}\right\} .
\end{array}
$$

Since $X \stackrel{p}{\rightarrow} X / G$ is a finite cover, $\widetilde{X}=\widetilde{X / G}$. Suppose that $X / G$ is a Jiang space. Given $\alpha \in \pi_{1}(X) \equiv \operatorname{Cov}(\eta: \widetilde{X} \rightarrow X)$, we identify $\alpha$ with $\bar{\alpha}=p_{\sharp}(\alpha) \in \pi_{1}(X / G) \equiv \operatorname{Cov}(p \circ \eta)$. There exists a cyclic homotopy $\left\{\bar{h}_{t}\right\}: 1_{X / G} \sim 1_{X / G}$ which lifts to $\left\{\tilde{h}_{t}\right\}: 1_{\bar{X}} \sim \bar{\alpha}(=\alpha)$. Project $\left\{\tilde{h}_{t}\right\}$ on $X$ to obtain a cyclic homotopy $\left\{h_{t}\right\}: 1_{X} \sim 1_{X}$. It follows from the Covering Homotopy Theorem that $\left\{h_{t}\right\}$ is a $G$-homotopy. 
Since the lens space $L_{q}^{2 n-1}$ is a Jiang space and is the orbit space of $S^{2 n-1}$ under the free $\mathbf{Z}_{q}$ action, any odd dimensional sphere $S^{2 n-1}$ is a $\mathbf{Z}_{q}$-Jiang space. More generally, for any positive relatively prime integers $p, q, \mathbf{Z}_{p}$ acts freely on $L_{q}^{2 n-1}$ with orbit space $L_{p q}^{2 n-1}$. Hence by $4.9, L_{q}^{2 n-1}$ is a $\mathbf{Z}_{p}$-Jiang space.

THEOREM 4.10. Let $G$ be a compact Lie group and $X$ be a compact $G$-ENR. For each $(H) \in \mathscr{F}$, we assume that $X^{H}$ is connected. Suppose that $X$ satisfies the G-Jiang condition. For any G-map $f: X \rightarrow X$ and $(H) \in \mathscr{F}$, either

(1) $L\left(f^{H}\right)=0 \Rightarrow N_{W H}\left(f^{H}\right)=0$, or

(2) $L\left(f^{H}\right) \neq 0 \Rightarrow N_{W H}\left(f^{H}\right)=\# R\left(\varphi_{W H}, \pi_{1}\left(X^{H}\right)\right)<\infty$, where $L\left(f^{H}\right)$ is the Lefschetz number of $f^{H}: X^{H} \rightarrow X^{H}$.

Proof. Let $(H) \in \mathscr{F}$ and $\tilde{f}^{H}$ be a lift of $f^{H}$. Suppose that $N_{1}=W H\left(p_{H}\right.$ Fix $\left.\tilde{f}^{H}\right)$ and $N_{2}=W H\left(p_{H}\right.$ Fix $\left.\alpha \tilde{f}^{H}\right)$ are two distinct $W H$-fixed point classes. Since $X$ is a $G$-Jiang space, there exists a $W H$-cyclic homotopy $\left\{h_{t}\right\}: 1_{X^{H}} \sim 1_{X^{H}}$ which lifts to $\left\{\tilde{h}_{t}\right\}: 1_{\tilde{X}^{H}} \sim \alpha$. Hence there exists a homotopy $\tilde{f}^{H} \sim \alpha \tilde{f}^{H}$ covering a $W H$-cyclic homotopy $f^{H} \sim f^{H}$. It follows from $[\mathbf{J}, \mathbf{I} .3 .10]$ that $I\left(f^{H}, N_{1}\right)=$ $I\left(f^{H}, N_{2}\right)$. Since $\alpha$ is arbitrary, we conclude that all $W H$-fpcs have the same index. By the normalization property of the Lefschetz number, $L\left(f^{H}\right)=\sum I\left(f^{H}, N\right)$ where $N$ varies over the set of $W H$-fpcs. Thus, if $L\left(f^{H}\right)=0$ then every $W H$-fpc is inessential. If $L\left(f^{H}\right) \neq 0$ then every $W H$-fpc is essential and hence by 4.5 , $N_{W H}\left(f^{H}\right)=\# R\left(\varphi_{W H}, \pi_{1}\left(X^{H}\right)\right)$.

REMARK 4.11. Note that if $N_{W H}\left(f^{H}\right)=0$ then the ordinary Nielsen number $N\left(f^{H}\right)=0$. Furthermore, a compact $G$-manifold $X$ such that $X^{H}$ is 1 -connected for $(H) \in \mathscr{F}$ is a $G$-Jiang space. Thus we can deduce the main results in [Wi] and [V] from 4.10(1) together with [FW].

In $[\mathbf{K}]$, Komiya showed that the fixed point index of $f^{(H)}: X^{(H)} \rightarrow$ $X^{(H)}$ is given by

$$
l\left(f^{(H)}\right)=\sum_{(H) \leq(K)} \chi(G / K) \cdot a_{(K)}(f)
$$

for some integers $\left\{a_{(K)}(f)\right\}$.

In the case where $G$ is a finite abelian group and $X$ is compact (*) 
reduces to

$(* *)$

$$
L\left(f^{H}\right)=\sum_{(H) \leq(K) \in \operatorname{Iso}(X)}[G: K] \cdot a_{(K)}(f) .
$$

Together with 4.10 , we obtain

COROLlaRY 4.12. Let $G$ be a finite abelian group and $X$ be a compact G-ENR such that for each $(H) \in \operatorname{Iso}(X), X^{H}$ is connected. If $X$ satisfies the $G$-Jiang condition, then for any $G$-map $f: X \rightarrow X$ and $(H) \in \operatorname{Iso}(X)$, there exist integers $I_{(H)}(f)$ such that

$$
N_{W H}\left(f^{H}\right) \cdot I_{(H)}(f)=\sum_{(H) \leq(K) \in \operatorname{Iso}(X)}[G: K] \cdot a_{(K)}(f)
$$

and

$$
a_{(K)}(f)=\frac{1}{[G: K]} \sum_{(K) \leq(H) \in \operatorname{Iso}(X)} \mu((K),(H)) N_{W H}\left(f^{H}\right) \cdot I_{(H)}(f)
$$

where $\mu($,$) is the Möbius function on \operatorname{Iso}(X)$.

Proof. Since $X$ is a $G$-Jiang space, by 4.10 , all $W H$-fpcs are either inessential or essential of the same index. We then have $L\left(f^{H}\right)=$ $N_{W H}\left(f^{H}\right) \cdot I_{(H)}(f)$ for some integer $I_{(H)}(f)$ which is 0 if $L\left(f^{H}\right)=0$. The first equality is then established from $(* *)$. The second equality follows from the Möbius Inversion formula (see [A]).

We conclude this section by relating some of the equivariant Nielsen invariants to the ordinary Nielsen numbers $\left\{N\left(f^{H}\right)\right\}$.

Recall in 4.4 the Reidemeister action of $\widetilde{W}$ on $\pi$ via $\alpha \mapsto$ $\tilde{\gamma} \alpha \varphi_{W}(\tilde{\gamma})^{-1}$. Let $T_{W}(\alpha)=\left\{\tilde{\gamma} \in \widetilde{W} \mid \tilde{\gamma} \alpha \varphi_{W}(\tilde{\gamma})^{-1}=\alpha\right\}$ be the subgroup of elements that act trivially on $\pi$.

LEMMA 4.13. Let $\alpha \in \pi$. Suppose that $T_{W}(\alpha) \subset \pi$. If $\beta=$ $\tilde{\gamma} \alpha \varphi_{W}(\tilde{\gamma})^{-1}$, then $\langle\alpha\rangle \neq\langle\beta\rangle$ unless $\tilde{\gamma} \in \pi$ where $\langle\delta\rangle$ denotes the ordinary Reidemeister class of $\delta \in \pi$.

Proof. If $\beta=\sigma \alpha\left(\varphi_{W} \mid \pi\right)(\sigma)^{-1}$ for some $\sigma \in \pi$ then

$$
\begin{array}{r}
\tilde{\gamma} \alpha \varphi_{W}(\tilde{\gamma})^{-1}=\sigma \alpha \varphi_{W}(\sigma)^{-1} \\
\Rightarrow \alpha=\left(\sigma^{-1} \tilde{\gamma}\right) \alpha \varphi_{W}\left(\sigma^{-1} \tilde{\gamma}\right)^{-1} \\
\Rightarrow \sigma^{-1} \tilde{\gamma} \in T_{W}(\alpha) \subset \pi \\
\Rightarrow \tilde{\gamma} \in \pi .
\end{array}
$$


THEOREM 4.14. Let $G$ be a finite abelian group and $X$ be a compact $G$-ENR such that $X^{H}$ is connected for every $(H) \in \operatorname{Iso}(X)$. Let $f: X \rightarrow X$ be a G-map. Suppose that for each $(H) \in \operatorname{Iso}(X)$, $J\left(X^{H}\right)=\pi_{1}\left(X^{H}\right), T_{W H}(\sigma) \subset \pi_{1}\left(X^{H}\right), \forall \sigma \in \pi_{1}\left(X^{H}\right), L\left(f^{H}\right) \neq 0$ and $\tau_{(H) \leq(K)}$ is injective for all $(H) \leq(K) \in \operatorname{Iso}(X)$. Then for each $(H) \in \operatorname{Iso}(X)$,

$$
[G: H] \cdot N O_{G}\left(f_{H}\right)=N_{G}\left(f_{H}\right)=\sum_{(H) \leq(K) \in \operatorname{Iso}(X)} \mu((H),(K)) N\left(f^{K}\right) .
$$

Proof. Since $G$ is abelian, $X_{(H)}=X_{H}$ and $X^{H}=\bigsqcup_{(H) \leq(K)} X_{K}$ for $(H) \in \operatorname{Iso}(X)$. Since every $W K$-fpc is a disjoint union of ordinary fixed point classes, $J\left(X^{K}\right)=\pi_{1}\left(X^{K}\right)$ and $L\left(f^{K}\right) \neq 0$ imply that all $W K$-fpcs are essential. From the Definition 2.6, $N O_{G}\left(f_{K}\right)=$ $N_{G}\left(f_{K}\right) /|W K|=N_{G}\left(f_{K}\right) /[G: K]$. Therefore, Fix $f^{H}=\bigsqcup_{(H) \leq(K)}$ Fix $f_{K}$ and Fix $f_{K}$ is the union of exactly $N O_{G}\left(f_{K}\right)$ many $W K$-fpcs. By 4.13, each $W K$-fpc is a disjoint union of $|W K|=[G: K]$ ordinary fixed point classes. Furthermore, since $\tau_{(K) \leq(H)}$ injects for all $(K) \leq(H) \in \operatorname{Iso}(X)$, we conclude that any two ordinary fixed point classes of $f^{H}$ from two distinct $W H$-fpcs must be contained in two distinct ordinary fixed point classes of $f^{K}$. Thus,

$$
N\left(f^{K}\right)=\sum_{(K) \leq(H)} N O_{G}\left(f_{H}\right) \cdot[G: H]=\sum_{(K) \leq(H)} N_{G}\left(f_{H}\right) .
$$

Applying the Möbius inversion formula to the equality above completes the proof.

REMARK 4.15. Since each $X^{H}$ is a Jiang space,

$$
N\left(f^{H}\right)=\# \operatorname{Coker}\left(1-f_{\sharp}^{H}\right)
$$

where $f_{\sharp}^{H}: \pi_{1}\left(X^{H}\right) \rightarrow \pi_{1}\left(X^{H}\right)$ is the induced homomorphism. Thus, the formula in 4.14 can be written as

$$
[G: H] \cdot N O_{G}\left(f_{H}\right)=N_{G}\left(f_{H}\right)=\sum_{(H) \leq(K)} \mu((H),(K)) \cdot \# \operatorname{Coker}\left(1-f_{\sharp}^{K}\right) \text {. }
$$

EXAMPLE 4.16. Consider the $G$-space in Example 3.9 as $T^{5} \times S^{2}$ Here let $X=T^{5}$ be the first component with the same $G=\mathbf{Z}_{6}$ action as before. Let $f: X \rightarrow X$ be given by

$$
f\left(e^{i \theta_{1}}, \ldots, e^{i \theta_{5}}\right)=\left(e^{i 2 \theta_{2}}, e^{i 2 \theta_{1}}, e^{-i \theta_{5}}, e^{-i \theta_{3}}, e^{-i \theta_{4}}\right)
$$


with

$$
\text { Fix } f=\left\{\left(\omega, \omega^{2}, \pm(1,1,1)\right) \mid \omega^{3}=1\right\} .
$$

Note that $N(f)=6, N\left(f^{\langle\alpha\rangle}\right)=2, N\left(f^{\langle\beta\rangle}\right)=6$ and $N\left(f^{G}\right)=2$. One can easily check that for each $(H) \in \operatorname{Iso}(X), X^{H}$ is a Jiang space, $L\left(f^{H}\right) \neq 0$ and $\tau_{(H) \leq(K)}$ is injective for all $(H) \leq(K) \in \operatorname{Iso}(X)$. Furthermore, $T_{W H} \subset \pi_{1}\left(X^{H}\right)$ for $H \in \operatorname{Iso}(X)$. By 4.14,

$$
N_{G}\left(f_{H}\right)=\sum_{(H) \leq(K) \in \operatorname{Iso}(X)} \mu((H),(K)) N\left(f^{K}\right) .
$$

For a finite cyclic group $G$ and $(H) \leq(K) \in \operatorname{Iso}(X), \mu((H),(K))=$ $\mu(|K / H|)$ where $\mu(n)$ is the number theoretic Möbius function of a positive integer $n$. Hence,

$$
\begin{aligned}
N_{G}\left(f_{G}\right) & =\mu(1) N\left(f^{G}\right)=N\left(f^{G}\right)=2 ; \\
N_{G}\left(f_{\langle\alpha\rangle}\right) & =N\left(f^{\langle\alpha\rangle}\right)+\mu(3) N\left(f^{G}\right)=2-2=0 ; \\
N_{G}\left(f_{\langle\beta\rangle}\right) & =N\left(f^{\langle\beta\rangle}\right)+\mu(2) N\left(f^{G}\right)=6-2=4 ; \\
N_{G}\left(f_{(1)}\right) & =N(f)+\mu(2) N\left(f^{\langle\alpha\rangle}\right)+\mu(3) N\left(f^{\langle\beta\rangle}\right)+\mu(6) N\left(f^{G}\right) \\
& =6-2-6+2=0 .
\end{aligned}
$$

See [HPY, 3.8] and $\S 5$ for applications to periodic points.

5. Periodic points. We now relate the equivariant Nielsen theory developed thus far to the Nielsen theory of periodic points developed in [HPY], [HY] and [J]. We also outline a proof of a result announced in [Ha] (also [J, III.4.14]) concerning the minimality of certain Nielsen type numbers for periodic points.

Let $X$ be a compact connected ENR and $f: X \rightarrow X$ be a self map. For any positive integer $n$, let

$$
Y_{n}=X \times \cdots \times X \quad(n \text {-fold product }) .
$$

The cyclic group $\mathrm{Z}_{n}=\langle\zeta\rangle$ acts on $Y_{n}$ via

$$
\zeta \cdot\left(x_{1}, \ldots, x_{n}\right)=\left(x_{n}, x_{1}, \ldots, x_{n-1}\right), \quad x_{i} \in X .
$$

We associate to $f$ a $Z_{n}$-map $g_{f}: Y_{n} \rightarrow Y_{n}$ defined by

$$
g_{f}\left(x_{1}, \ldots, x_{n}\right)=\left(f\left(x_{n}\right), f\left(x_{1}\right), \ldots, f\left(x_{n-1}\right)\right) .
$$

For any positive integer $m$ with $m \mid n$,

$$
Y_{n}^{\mathbf{Z}_{m}} \approx X \times \cdots \times X
$$

$(n / m$-fold product)

and

$$
\text { Fix } g_{f}^{\mathbf{Z}_{m}}=\left\{\left(x, f(x), \ldots, f^{n / m-1}(x)\right) \mid x \in \operatorname{Fix} f^{n / m}\right\}
$$


It is easy to see that there is a 1-1 correspondence between Fix $g_{f}$ and Fix $f^{n}$. In particular, an $f$-orbit of a periodic point $x$ of period $n$ (i.e., $\left\{x, f(x), \ldots, f^{n-1}(x)\right\}, x \in$ Fix $f^{n}$ ) corresponds to the $\mathbf{Z}_{n^{-}}$ fixed orbit of the fixed point $\left(x, f(x), \ldots, f^{n-1}(x)\right)$ of $g_{f}$. Recall from [J, III. $\S 4$ ], a periodic point class of period $n$ is a fixed point class of $f^{n}$. A set of periodic point classes (of diverse periods) is said to be $f$-invariant if it is a union of $f$-orbits. In fact, there is a 1-1 correspondence between the set of nonempty $f$-orbits of periodic point classes and the set of nonempty $\mathbf{Z}_{n}$-fpcs of $g_{f}$ (here $W H=\mathbf{Z}_{n}$, $H=(1))$. To see this, let $\tilde{f}: \widetilde{X} \rightarrow \widetilde{X}$ be a lift of $f$ where $p: \widetilde{X} \rightarrow X$ is the universal cover. Let $\tilde{f}^{n}=\tilde{f} \circ \cdots \circ \tilde{f}$ ( $n$-copies) be the lift of $f^{n}$ so that every periodic point class of period $n$ is of the form $p$ Fix $\alpha \tilde{f}^{n}$ for some $\alpha \in \pi$ where $\pi=\operatorname{Cov}(p) \equiv \pi_{1}(X)$. Consider the lift $\tilde{g}_{f}$ of $g_{f}$ given by

$$
\tilde{g}_{f}\left(\tilde{x}_{1}, \ldots, \tilde{x}_{n}\right)=\left(\tilde{f}\left(\tilde{x}_{n}\right), \tilde{f}\left(\tilde{x}_{1}\right), \ldots, \tilde{f}\left(\tilde{x}_{n-1}\right)\right), \quad \tilde{x}_{i} \in \tilde{X} .
$$

We have the following commutative diagrams of liftings

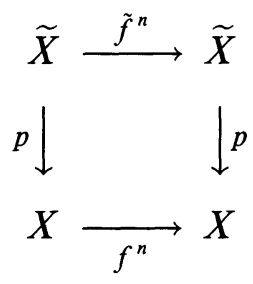

and

$$
\begin{array}{cc}
\tilde{X} \times \cdots \times \widetilde{X}=\widetilde{Y}_{n} \stackrel{\tilde{g}_{f}}{\longrightarrow} \widetilde{Y}_{n} \\
\eta \quad \\
X \times \cdots \times X=Y_{n} \underset{g_{f}}{\longrightarrow} Y_{n}
\end{array}
$$

Let

$$
\begin{aligned}
\mathscr{O}\left(f^{n}\right) & =\{f \text {-orbits of periodic point classes of period } n\} \\
& =\left\{S \mid S=\left\{p \operatorname{Fix} \alpha \tilde{f}^{n}, f\left(p \operatorname{Fix} \alpha \tilde{f}^{n}\right), \ldots, f^{n-1}\left(p \operatorname{Fix} \alpha \tilde{f}^{n}\right)\right\}\right\} .
\end{aligned}
$$

If $S$ is the $f$-orbit of $p$ Fix $\alpha \tilde{f}^{n}$ for some $\alpha \in \pi$, we associate it to the $\mathbf{Z}_{n}$-fpc $\mathbf{Z}_{n}\left(\eta\right.$ Fix $\left.\alpha_{\varphi} \tilde{g}_{f}\right) \in \operatorname{FPC}_{\mathbf{Z}_{n}}\left(g_{f}\right)$ where $\alpha_{\varphi}=1 \times \cdots \times 1 \times \alpha \in$ $\operatorname{Cov}(\eta) \equiv \pi \times \cdots \times \pi, \varphi: \pi \rightarrow \pi$ is the homomorphism corresponding to $\tilde{f}$. Let $\varphi^{(k)}(\alpha)=\varphi \circ \cdots \circ \varphi(\alpha) \quad(k$ copies $)$. 
Proposition 5.1. There is a function $\psi_{n}: \mathscr{O}\left(f^{n}\right) \rightarrow \mathrm{FPC}_{\mathbf{Z}_{n}}\left(g_{f}\right)$ given by

$$
\psi_{n}\left(\mathscr{O}\left(p \operatorname{Fix} \alpha \tilde{f}^{n}\right)\right)=\mathbf{Z}_{n}\left(\eta \operatorname{Fix} \alpha_{\varphi} \tilde{g}_{f}\right)
$$

where $\mathscr{O}\left(p\right.$ Fix $\left.\alpha \tilde{f}^{n}\right)=f$-orbit of $p$ Fix $\alpha \tilde{f}^{n}$.

Furthermore, the restriction

$$
\psi_{n}^{c}:\left\{S \in \mathscr{O}\left(f^{n}\right) \mid S \neq \varnothing\right\} \rightarrow\left\{N \in \mathrm{FPC}_{\mathbf{Z}_{n}}\left(g_{f}\right) \mid N \neq \varnothing\right\}
$$

is a bijection.

Proof. Let $S \in \mathscr{O}\left(f^{n}\right)$ and $p$ Fix $\alpha \tilde{f}^{n} \in S$. It follows from [J], III.3.3] that every element of $S$ has the form $p$ Fix $\beta \tilde{f}^{n}=f^{k} p$ Fix $\alpha \tilde{f}^{n}$ for some $1 \leq k \leq n$. To show that $\psi_{n}$ is well-defined, we need to show that if $p \operatorname{Fix} \alpha \tilde{f}^{n}$ and $p \operatorname{Fix} \beta \tilde{f}^{n} \in S$ then $\mathbf{Z}_{n}\left(\eta\right.$ Fix $\left.\alpha_{\varphi} \tilde{g}_{f}\right)=$ $\mathbf{Z}_{n}\left(\eta\right.$ Fix $\left.\beta_{\varphi} \tilde{g}_{f}\right)$, i.e., $\exists \tilde{\gamma} \in \widetilde{\mathbf{Z}_{n}}$ such that $\beta_{\varphi}=\tilde{\gamma} \alpha_{\varphi} \varphi_{\mathbf{Z}_{n}}(\tilde{\gamma})^{-1}$ (cf. 4.5).

Note that $\mathbf{Z}_{n}$ acts on $\tilde{Y}_{n}$ via $\left(\tilde{x}_{1}, \ldots, \tilde{x}_{n}\right) \mapsto\left(\tilde{x}_{n}, \tilde{x}_{1}, \ldots, \tilde{x}_{n-1}\right)$ so that the group $\widetilde{Z_{n}}$ (cf. $\S 2$ ) covering the $\mathbf{Z}_{n}$-action on $Y_{n}$ is the semi-direct product of $\pi \times \cdots \times \pi$ and $\mathbf{Z}_{n}$. Furthermore, the homomorphism $\varphi_{\mathbf{Z}_{n}}: \widetilde{\mathbf{Z}_{n}} \rightarrow \widetilde{\mathbf{Z}_{n}}$ corresponding to the lift $\tilde{g}_{f}$ is given by

$$
\varphi_{\mathbf{Z}_{n}}\left(\left(\alpha_{1} \times \cdots \times \alpha_{n}\right) \cdot \zeta^{k}\right)=\left(\varphi\left(\alpha_{n}\right) \times \varphi\left(\alpha_{1}\right) \times \cdots \times \varphi\left(\alpha_{n-1}\right)\right) \cdot \zeta^{k}
$$

where $\zeta$ is the generator of $\mathbf{Z}_{n}$.

Suppose that $f^{k}\left(p\right.$ Fix $\left.\alpha \tilde{f}^{n}\right)=p \operatorname{Fix} \beta \tilde{f}^{n}$ for some $1 \leq k \leq n$. Then following [J, III.3.3] we have

$$
\begin{aligned}
p \operatorname{Fix} \beta \tilde{f}^{n} & =f^{k}\left(p \operatorname{Fix} \alpha \tilde{f}^{n}\right)=p \tilde{f}^{k}\left(\operatorname{Fix} \alpha \tilde{f}^{n}\right)=p \operatorname{Fix} \tilde{f}^{k} \alpha \tilde{f}^{n-k} \\
& =p \operatorname{Fix} \varphi^{(k)}(\alpha) \tilde{f}^{k} \tilde{f}^{n-k}=p \operatorname{Fix} \varphi^{(k)}(\alpha) \tilde{f}^{n} .
\end{aligned}
$$

Thus there exists $\gamma \in \pi$ such that $\beta=\gamma \varphi^{(k)}(\alpha) \varphi^{(n)}(\gamma)^{-1}$. Hence,

$$
\beta_{\varphi}=1 \times \cdots \times 1 \times \gamma \varphi^{(k)}(\alpha) \varphi^{(n)}(\gamma)^{-1} .
$$

Let $\hat{\gamma}=\left(\gamma \times \cdots \times \varphi^{(n-1)}(\gamma)\right) \cdot \zeta^{n-1}=\left(\varphi(\gamma) \times \cdots \times \varphi^{(n-1)}(\gamma) \times \gamma\right)$. Then

$$
\begin{gathered}
\hat{\gamma}\left(\varphi^{(k)}(\alpha)\right)_{\varphi} \varphi_{\mathbf{Z}_{n}}(\hat{\gamma})^{-1}=\hat{\gamma}\left(1 \times \cdots \times 1 \times \varphi^{(k)}(\alpha)\right) \varphi_{\mathbf{Z}_{n}}(\hat{\gamma})^{-1} \\
=\left(1 \times \cdots \times 1 \times \gamma \varphi^{(k)}(\alpha) \varphi^{(n)}(\gamma)^{-1}\right)=\beta_{\varphi} .
\end{gathered}
$$

It follows then that $\left(\varphi^{(k)}(\alpha)\right)_{\varphi}=\zeta^{k}\left(1 \times \cdots \times \varphi^{(k)}(\alpha) \times \cdots \times 1\right) \varphi_{\mathbf{Z}_{n}}\left(\zeta^{k}\right)^{-1}$ and by applying $\varphi_{\mathbf{Z}_{n}} k$ times to $\alpha_{\varphi},\left(1 \times \cdots \times \varphi^{(k)}(\alpha) \times \cdots \times 1\right)$ is $\varphi_{\mathrm{Z}_{n}}$-conjugate to $\alpha_{\varphi}$.

Let

$$
\mathscr{O}^{c}\left(f^{n}\right)=\left\{S \in \mathscr{O}\left(f^{n}\right) \mid S \neq \varnothing\right\}
$$


and

$$
\mathrm{FPC}_{\mathbf{Z}_{n}}^{c}\left(g_{f}\right)=\left\{N \in \mathrm{FPC}_{\mathbf{Z}_{n}}\left(g_{f}\right) \mid N \neq \varnothing\right\} .
$$

We will make use of the geometric characterization of nonempty fixed point classes in the classical sense ([Br] or $[\mathbf{J}]$ ) and in 2.4. If $\left(x, f(x), \ldots, f^{n-1}(x)\right)$ and $\left(y, f(y), \ldots, f^{n-1}(y)\right)$ belong to the same $\mathbf{Z}_{n}$-fpc then either they belong to the same $\mathbf{Z}_{n}$-orbit in which case, $y=f^{i}(x)$ for some $i$ or $y$ is Nielsen equivalent to $f^{j}(x)$ for some $j$. In both cases, $x$ and $y$ belong to the same $f$-orbit of a periodic point class. This shows that $\psi_{n}^{c}$ is surjective.

Let $S_{1} \neq S_{2} \in \mathscr{O}^{c}\left(f^{n}\right)$ and $x_{1}, x_{2}$ be fixed points in $S_{1}$ and $S_{2}$ respectively. Since $S_{1}$ and $S_{2}$ are distinct, $x_{1}$ and $x_{2}$ cannot be Nielsen equivalent. That they do not belong to the same $f$-orbits implies that $\left(x_{1}, \ldots, f^{n-1}\left(x_{1}\right)\right)$ and $\left(x_{2}, \ldots, f^{n-1}\left(x_{2}\right)\right)$ must lie in distinct $\mathbf{Z}_{n}$-orbits. Thus $\psi_{n}^{c}$ is injective.

REMARK 5.2. A class $N \in \mathrm{FPC}_{\mathbf{Z}_{n}}^{c}\left(g_{f}\right)$ does not contain any $\mathbf{Z}_{m}$-fpc for any $m, m \mid n$ iff $\psi_{n}^{-1}(N) \in \mathscr{O}^{c}\left(f^{n}\right)$ is the $f$-orbit of a nonempty periodic point class of period $n$ which does not contain any periodic point class of period $m$. A periodic point class of period $n$ is essential iff the $f$-orbit containing it corresponds under $\psi_{n}$ to an essential $\mathbf{Z}_{n^{-}}$ fpc. Thus, there is a 1-1 correspondence between the set of $f$-orbits of irreducible (i.e., does not contain any periodic point class of period $m<n$ ) essential periodic point classes of period $n$ and the set of essential $\mathbf{Z}_{n}$-fpcs of $g_{f}$.

Recall from [ $\mathbf{J}]$ that the height of an $f$-invariant set of periodic point classes is the sum of the periods of the $f$-orbits in the set. The Nielsen type number of period $n$ is given by

$$
\begin{aligned}
N P_{n}(f)= & \text { the height of the set of irreducible } \\
& \text { essential periodic point classes of period } n .
\end{aligned}
$$

Proposition 5.3.

$$
N P_{n}(f)=N_{\mathbf{Z}_{n}}\left(\left(g_{f}\right)_{(1)}\right)
$$

where (1) is the trivial subgroup of $\mathbf{Z}_{n}$.

Proof. The set of irreducible essential periodic point classes contains only periodic points of least period $n=\left|Z_{n}\right|$ while the corresponding set of essential $Z_{n}$-fpcs lie in $Y_{n_{(1)}}$. The assertion follows from the definitions of $N P_{n}(f)$ and $N_{\mathbf{Z}_{n}}\left(\left(g_{f}\right)_{(1)}\right)$ and Remark 5.2 above. 
The Nielsen type number for the nth iterate is given by

$N F_{n}(f)=\min$ \{height of $Q \mid Q$ is $f$-invariant and every

essential periodic point class of period $m$

with $m \mid n$ contains at least one class from $Q\}$.

We also let

$$
\begin{aligned}
P_{n}(f) & =\operatorname{Fix} f^{n}-\bigcup_{m<n} \operatorname{Fix} f^{m}, \\
M P_{n}[f] & =\min \left\{\# P_{n}(g) \mid g \sim f\right\} \quad \text { and } \\
M F_{n}[f] & =\min \left\{\# \operatorname{Fix} g^{n} \mid g \sim f\right\} .
\end{aligned}
$$

REMARK 5.4. First observe that

$$
M P_{n}[f] \geq M_{\mathbf{Z}_{n}}\left(\left(g_{f}\right)_{(1)}\right) \quad \text { (cf. 2.8) }
$$

and

$$
M F_{n}[f] \geq m_{\mathbf{Z}_{n}}\left(\left(g_{f}\right)^{(1)}\right) \quad \text { (cf. 3.6) }
$$

The minimality Theorem 3.3 together with 5.3 imply that

$$
N P_{n}(f)=N_{\mathbf{Z}_{n}}\left(\left(g_{f}\right)_{(1)}\right)=M_{\mathbf{Z}_{n}}\left(\left(g_{f}\right)_{(1)}\right) \leq M P_{n}[f] .
$$

These inequalities may not be equalities since $g_{f} \sim_{G} \varepsilon$ does not guarantee that $\varepsilon$ is of the form $g_{h}$ for some $h \sim f$.

THeOREM 5.5 [Ha], [J, III.4.14]. Let $X$ be a compact connected smooth manifold of $\operatorname{dim} X \geq 5$. For any self map $f: X \rightarrow X$ and for any positive integer $n$,

(1) $M P_{n}[f]=N P_{n}(f)$,

(2) $M F_{n}[f]=N F_{n}(f)$.

Sketch of Proof. By the approximation theorem [J, III.§3 Appendix], we may assume without loss of generality that \# Fix $f^{n}<\infty$ and thus \# Fix $g_{f}<\infty$. Let $x, y \in$ Fix $f^{n}$ such that $x$ and $y$ are in the same periodic point class but not in the same $f$-orbit. In other words, $\mathbf{x}=\left(x, f(x), \ldots, f^{n-1}(x)\right)$ and $\mathbf{y}=\left(y, f(y), \ldots, f^{n-1}(y)\right)$ belong to the same $\mathbf{Z}_{n}$-fpc but not in the same $\mathbf{Z}_{n}$-orbit. If $\alpha:[0,1] \rightarrow X$ is a path such that $\alpha(0)=x, \alpha(1)=y$ and $\alpha \sim_{h_{t}} f^{n} \alpha$ (rel endpoints) then

$$
\vec{\alpha} \sim_{\mathbf{h}_{t}} g_{f} \vec{\alpha} \quad \text { (rel endpoints) }
$$

where $\vec{\alpha}=\left(\alpha, f \alpha, \ldots, f^{n-1} \alpha\right)$ is a path in $Y_{n}$ from $\mathbf{x}$ to $\mathbf{y}$ and $\mathbf{h}_{t}=\left(h_{t}, f \alpha, \ldots, f^{n-1} \alpha\right)$. The proof of 3.1 suggests that we alter $f$ 
inside a small euclidean neighborhood of $\alpha$ by employing the classical Wecken method as in [Br, VIII.C] so that we can unite $f^{i}(x)$ and $f^{i}(y)$ simultaneously for all $i$. The crucial fact (e.g. [Br, VIII.C.6 p. 139]) is that $\left\{f^{n}\left(\zeta^{k} \alpha\right)\left(\zeta^{k} \alpha\right)^{-1}\right\}$ be trivial in $\pi_{1}\left(Y_{n}\right)=\pi_{1}(X) \times \cdots \times$ $\pi_{1}(X)$. This is precisely the case when $\operatorname{dim} X \geq 5$ because any two loops are unlinked by general position. Hence the resulting $\mathbf{Z}_{n}$-map in 3.1 can be taken in the form of $g_{h}$ for some $h \sim f$ relative to the complement of a small neighborhood of $\alpha \cup \cdots \cup f^{n-1} \alpha$. Then (1) follows from the same argument as in 3.3. For (2), we can find $h \sim f$ such that

by 3.7. Thus,

$$
\# \text { Fix } g_{h}=m_{\mathbf{Z}_{n}}\left(\left(g_{f}\right)^{(1)}\right)
$$

$$
M F_{n}[f]=m_{\mathbf{Z}_{n}}\left(\left(g_{f}\right)^{(1)}\right) .
$$

Let $Q=$ Fix $g_{h}$. It is clear that $Q$ is a union of $f$-orbits of periodic point classes of diverse periods and that every essential periodic point class of period $m$ with $m \mid n$ contains at least one class from $Q$. Moreover, height $(Q)=\#$ Fix $g_{h}$ and thus (2) follows.

6. Concluding remarks. In developing the equivariant Nielsen theory in this paper, we restrict ourselves to compact $G$-ENRs $X$ and $G$-maps $f: X \rightarrow X$. We can extend easily to the case $f: V \rightarrow X$ where $V$ is an open $G$-invariant subset of $X$ and Fix $f$ is compact in $V$ by considering a restricted class of $G$-homotopies (e.g. [D4], [K] and [W2]). We may also generalize in another direction to compact $G$-ANRs (e.g. [HPY], [HY]). Furthermore, we may relax the assumptions on $X^{H}$ by considering connected components $X_{c}^{H}$ such that $f \mid X_{c}^{H}: X_{c}^{H} \rightarrow X_{c}^{H}$.

We showed in $\S 5$ how the Nielsen theory for periodic points is related to the equivariant theory. In particular, we established the equality $N P_{n}(f)=N_{\mathbf{Z}_{n}}\left(\left(g_{f}\right)_{(1)}\right)$ in 5.3. It is easy to see that, for example, 4.14 is the equivariant analog of [HPY, 3.7].

\section{REFERENCES}

[A] A. Aigner, Combinarorial Theory, Springer-Verlag, Heidelberg, 1979.

[B] G. Bredon, Introduction to Compact Transformation Groups, Academic Press, New York, 1972.

[Br] R. F. Brown, The Lefschetz Fixed Point Theorem, Scott Foresman, Illinois, 1971.

[tD] T. tomDieck, Transformation Groups, de Gruyter, Berlin, New York, 1987.

[D1] A. Dold, Lectures on Algebraic Topology (2nd ed.), Springer-Verlag, Heidelberg, 1980. 
[D2] _ Fixed point index and fuxed point theorems for euclidean neighborhood retracts, Topology, 4 (1965), 1-8.

[D3] _ - Fixed point indices of iterated maps, Invent. Math., 74 (1983), 419-435.

[D4] _ Fixed point theory and homotopy theory, Contemp. Math., vol. 12, Amer. Math. Soc., Providence, RI, 1982, pp. 105-115.

[FH] E. Fadell and S. Husseini, Fixed point theory for non-simply connected manifolds, Topology, 20 (1981), 53-92.

[FW] E. Fadell and P. Wong, On deforming G-maps to be fixed point free, Pacific J. Math., 132 (1988), 277-281.

[Ha] B. Halpern, The minimum number of periodic points, Abstracts Amer. Math. Soc., 1 (1980), 269.

[HPY] P. Heath, R. Piccinini, and C. You, Nielsen Type Numbers For Periodic Points I, Springer Lecture Notes in Math., vol. 1411, 1989, pp. 88-106.

[HY] P. Heath and C. You, Nielsen type numbers for periodic points, II, Topology and its Appl., 43 (1992), 219-236.

[J] B. Jiang, Lectures on Nielsen Fixed Point Theory, Contemp. Math., vol. 14, Amer. Math. Soc., 1982.

[K] K. Komiya, Fixed point indices of equivariant maps and Möbius inversion, Invent. Math., 91 (1988), 129-135.

[S] H. Schirmer, A relative Nielsen number, Pacific J. Math., 122 (1986), 459473.

[U] H. Ulrich, Fixed Point Theory of Parametrized Equivariant Maps, Springer Lecture Notes in Math., vol. 1343, 1988.

[V] A. Vidal, On equivariant deformation of maps, Publ. Mat., 32 (1988), 115121.

[Wi] D. Wilczyński, Fixed point free equivariant homotopy classes, Fund. Math., 123 (1984), 47-60.

[W1] P. Wong, On the location of fixed points of G-deformations, Topology Appl., 39 (1991), 159-165.

[W2] _ Equivariant Nielsen fixed point theory for G-maps, Pacific J. Math., 150 (1991), 179-200.

[Z] X. Zhao, A Relative Nielsen Number for the Complement, Springer Lecture Notes in Math., vol. 1411, 1989, pp. 189-199.

Received September 11, 1991 and in revised form January 6, 1992.

Bates College

LEWISTON, ME 04240

E-mail address: pwong@abacus.bates.edu 



\section{PACIFIC JOURNAL OF MATHEMATICS}

Founded by

E. F. BECKENBACH (1906-1982) F. Wolf (1904-1989)

\section{EDITORS}

\author{
V. S. VARADARAJAN \\ (Managing Editor) \\ University of California \\ Los Angeles, CA 90024-1555 \\ vsv@math.ucla.edu \\ F. Michael Christ \\ University of California \\ Los Angeles, CA 90024-1555 \\ christ@math.ucla.edu
}

\section{Herbert Clemens}

University of Utah

Salt Lake City, UT 84112

clemens@math.utah.edu

\author{
ThOMAs ENRIGHT \\ University of California, San Diego \\ La Jolla, CA 92093 \\ tenright@ucsd.edu \\ Nicholas Ercolani \\ University of Arizona \\ Tucson, AZ 85721 \\ ercolani@math.arizona.edu \\ R. FINN \\ Stanford University \\ Stanford, CA 94305 \\ finn@gauss.stanford.edu \\ VAUGHAN F. R. Jones \\ University of California \\ Berkeley, CA 94720 \\ vfr@math.berkeley.edu
}

STEVEN KeRCKHOFF

Stanford University

Stanford, CA 94305

spk@gauss.stanford.edu

Martin ScharlemanN

University of California

Santa Barbara, CA 93106

mgscharl@henri.ucsb.edu

Harold Stark

University of California, San Diego

La Jolla, CA 92093

\section{SUPPORTING INSTITUTIONS}

UNIVERSITY OF ARIZONA

UNIVERSITY OF BRITISH COLUMBIA

CALIFORNIA INSTITUTE OF TECHNOLOGY

UNIVERSITY OF CALIFORNIA

UNIVERSITY OF MONTANA

UNIVERSITY OF NEVADA, RENO

NEW MEXICO STATE UNIVERSITY

OREGON STATE UNIVERSITY
UNIVERSITY OF OREGON

UNIVERSITY OF SOUTHERN CALIFORNIA

STANFORD UNIVERSITY

UNIVERSITY OF HAWAII

UNIVERSITY OF UTAH

WASHINGTON STATE UNIVERSITY

UNIVERSITY OF WASHINGTON 


\section{PACIFIC JOURNAL OF MATHEMATICS}

Volume 159 No. $1 \quad$ May 1993

An application of the very weak Bernoulli condition for amenable groups

SCOT ROBERT ADAMS and JEFFREY EDWARD STEIF

An application of homogenization theory to harmonic analysis on solvable Lie groups of polynomial growth

G. Alexopoulos

The standard double soap bubble in $\mathbf{R}^{2}$ uniquely minimizes perimeter

Joel Foisy, Manuel Alfaro Garcia, JefFrey FARlowe

Brock, NiCKELOUS Hodges and JASON ZimbA

Pseudo regular elements and the auxiliary multiplication they induce

BARRY E. JOHNSON

A converse to a theorem of Komlós for convex subsets of $L_{1}$

CHRISTOPHER JOHN LENNARD

General Kac-Moody algebras and the Kazhdan-Lusztig conjecture

WAYNE L. NEIDHARDT

The flow space of a directed $G$-graph

WILLIAM LINDALL PASCHKE

Primitive ideals and derivations on noncommutative Banach algebras

MARK PHILLIP THOMAS

Equivariant Nielsen numbers

PETER N-S WONG

Volumes of tubular neighbourhoods of real algebraic varieties

RichaRd ALEXANDER WONGKEW

The intrinsic group of Majid's bicrossproduct Kac algebra 